\title{
A recommendation to perform a blood culture before the administration of intravenous antibiotics increased the detection of Staphylococcus aureus bacteremia
}

\author{
A. Jogenfors • L. Stark • J. Svefors • S. Löfgren • \\ B.-E. Malmvall • A. Matussek
}

Received: 7 August 2013 / Accepted: 25 October 2013 / Published online: 19 November 2013

(C) The Author(s) 2013. This article is published with open access at Springerlink.com

\begin{abstract}
In 2004, the Surviving Sepsis Campaign was launched to increase awareness and improve the outcome of severe sepsis. Accordingly, in Jönköping County, Sweden, a strong recommendation to perform a blood culture before the start of intravenous antibiotic treatment was introduced in 2007. Moreover, a reminder was included in the laboratory report to consult an infectious disease specialist when Staphylococcus aureus was isolated from a blood culture. Retrospectively, patients with at least one blood culture growing $S$. aureus during 2002 through 2003 (pre intervention $n=58$ ) or during 2008 through 2009 (post intervention $n=100$ ) were included. Medical records were evaluated regarding clinical data and outcome. Blood culture isolates were characterized by antibiotic susceptibility testing (AST) and S. aureus protein A (spa) gene typing. The annual incidence of $S$. aureus bacteremia (SAB) increased from 28 per 100,000 inhabitants at the pre intervention period to 45 per 100,000 at the post intervention period ( $p=0.046)$. During post intervention, the $\mathrm{SAB}$ incidence was significantly higher in men $(p=0.009)$.
\end{abstract}

\footnotetext{
A. Jogenfors

Faculty of Health Sciences, Linköping University, Linköping, Sweden

A. Jogenfors $\cdot$ L. Stark $\cdot$ S. Löfgren $\cdot$ A. Matussek $(\triangle)$

Department of Laboratory Services, Division of Medical Services, County Hospital Ryhov, Jönköping, Sweden

e-mail: Andreas.Matussek@lj.se

J. Svefors

Department of Infectious Diseases, County Hospital Ryhov, Jönköping, Sweden

B.-E. Malmvall

Futurum - The Academy for Healthcare, Jönköping County Council, Jönköping, Sweden
}

The mortality rate during hospital stay was $14 \%$ during pre intervention and $18 \%$ during post intervention $(p=0.47)$. The most common spa types were t012 and t084. The Surviving Sepsis Campaign resulted in an increased number of detected cases of SAB. The mortality rate was the same before and after the intervention, and no spa type correlated to certain clinical manifestations or mortality.

\section{Background}

Staphylococcus aureus bacteremia (SAB) correlates to sepsis, severe sepsis, and septic shock with high mortality [1]. An early diagnosis and a structured management and treatment of patients with $\mathrm{SAB}$ are essential to prevent a fatal progress [2, 3]. Guidelines for the management of severe sepsis and septic shock were introduced in 2004 [4]. The rate of methicillinresistant $S$. aureus (MRSA) in Sweden is low [5]; however, knowledge of the local prevalence of MRSA is crucial in the choice of empirical treatment [6].

In Jönköping County in the southern part of Sweden, a patient safety project aiming at the early identification and treatment of patients with suspected severe sepsis was introduced in 2007, in accordance with Dellinger et al. [4]. This included a strong recommendation to perform a blood culture before the start of an intravenous antibiotic treatment and a reminder in the laboratory report to consult an infectious disease specialist when $S$. aureus was isolated from a blood culture.

In this study, we investigate the incidence and outcome of SAB in Jönköping, Sweden, 2002 through 2003 and 2008 through 2009 to evaluate if the patient safety project resulted in an increase in SAB cases detected. Furthermore, we investigate the molecular epidemiology of $S$. aureus based on sequencing of the repeat region of the $S$. aureus protein A gene (spa typing) [7]. 


\section{Methods}

Setting

The study was performed at the County Hospital Ryhov in Jönköping, situated in the southern part of Sweden. The hospital has 638 beds and 3,500 employees. Inhabitants of the catchment area with an age above 18 years averaged 105 , 300 in the pre intervention period and 112,900 in the post intervention period.

\section{Study design}

This retrospective study is based on the evaluation of medical records from all patients $(n=158)$ with $\mathrm{SAB}$ at a pre intervention period of 2 years (2002 and 2003) $(n=58)$ and a post intervention period of 2 years (2008 and 2009) $(n=100)$ comparing medical history, clinical findings, and laboratory results. No independent evaluation of the clinical diagnosis was performed. For epidemiological purposes, spa typing of $S$. aureus in blood culture isolates was performed. In total, $163 \mathrm{~S}$. aureus isolates from 148 different patients were available for spa typing. To evaluate the long-term effect of the intervention, figures (number of blood cultures, number of patients with SAB, and annual incidence) from the 2 years 2011 and 2012 were included as a follow up.

\section{Intervention}

In 2004, new guidelines, aiming at an increased attention to patients at risk for severe sepsis, were introduced. The guidelines included a strong recommendation to always perform a blood culture before administrating the first intravenous antibiotic dose and a reminder in the laboratory report to consult an infectious disease specialist when $S$. aureus was isolated from a blood culture. This was implemented in Jönköping between 2003 and 2008, and, noteworthy, the nurse was committed to be responsible for taking a blood culture before starting the antibiotic treatment.

\section{Definitions}

SAB was defined as $S$. aureus obtained from one or both blood culture bottles. Severe sepsis was defined according to the American College of Chest Physicians/Society of Critical Care Medicine Consensus Conference Committee [8]. Nosocomial infections were defined as a positive blood culture taken later than $48 \mathrm{~h}$ after arrival to the hospital. The mortality was the allcause mortality. Recurrence was defined as a new episode of $\mathrm{SAB}$ within 1 year, but more than 4 weeks after the time of the first diagnosis. A relapse was defined as a recurrence caused by the same spa type as the primary episode and a reinfection was defined as a recurrence with a different spa type.
Antibiotic susceptibility testing

Antibiotic susceptibility testing (AST) was performed by the disk diffusion method on Iso-Sensitest Agar (Oxoid, Ltd., Basingstoke, UK) using specific antibiotic disks (Oxoid, Ltd., Basingstoke, UK) pre and post intervention according to the Swedish standard for AST (http://www.srga.org). The recommendations and antimicrobial susceptibility testing criteria of the Swedish Reference Group of Antibiotics (SRGA) were adhered to. The SRGA has, since 2002, adopted all recommendations on breakpoints and methodology from the European Committee on Antimicrobial Susceptibility Testing (EUCAST).

spa typing

The spa typing of blood culture isolates (stored in skimmed milk at $-80^{\circ} \mathrm{C}$ ) was performed as previously described [7, 9]. Sequencing was performed by GATC Biotech (GATC Biotech AG, Konstanz, Germany) and each isolate was assigned an spa type using the Ridom StaphType software (v1.5.21, Ridom GmbH, Würzburg, Germany). The Ridom StaphType software was also used to obtain based upon repeat pattern (BURP) clusters [10], enabling the determination of clonal relatedness. The default parameters $(x=5 ; y=4)$ were applied.

Panton-Valentine leukocidin testing

Panton-Valentine leukocidin (PVL) testing was performed on blood culture isolates from patients with pneumonia, according to Lina et al. [11].

\section{Statistical analyses}

IBM SPSS Statistics 19 and Microsoft Excel 2007 were used. A two-sided Chi-square test with one degree of freedom was used for the evaluation of proportions and the Mann-Whitney test was used for calculations of differences between means. A $p$-value $\leq 0.05$ was considered significant. Antibiotic-sensitive and -intermediate isolates were combined in statistical analyses.

Ethical considerations

This study complied with current Swedish laws.

\section{Results}

The number of blood cultures increased from 8,616 at pre intervention to 14,842 at post intervention $(72 \%, p<0.0001)$. The number of patients suffering from SAB during the pre intervention period was 58 and at post intervention it was 100 (73\%, $p=0.0037)$. The proportion of patients with SAB was 
the same during the pre and post intervention periods. The annual incidence of SAB increased from 28 per 100,000 inhabitants at pre intervention to 45 per 100,000 at post intervention $(p=0.046)$ (Table 1). At pre intervention, no statistically significant difference $(p=0.071)$ in the SAB rate was seen between women and men, whereas at post intervention, the SAB rate was significantly higher in men $(p=0.009)$. The number of blood cultures performed was significantly higher for men compared to women at pre $(p<0.0001)$ and post $(p<0.0001)$ intervention (Table 1$)$, and the increase of performed blood cultures was higher for men than for women $(p<0.0001)$.

The median age at SAB diagnosis was 69 and 75 years for men $(p=0.11)$ and 74 and 74 years for women $(p=0.33)$ during pre and post intervention, respectively. For men, the age-specific SAB rates were highest at 50-59 years of age at pre intervention and 80-89 years at post intervention. For women, the corresponding figures were 70-79 years at pre intervention and 50-59 years at post intervention (Fig. 1). At follow up, a further increase $(p<0.0001)$ in the number $(17$, 783) of blood cultures performed was noted, as well as a sustained rate $(p=0.35)$ of patients with SAB $(n=115)$ and annual incidence of 44 per 100,000 $(p=0.92)$.

The mortality among SAB patients during hospital stay was 14 and $18 \%$ at pre and post intervention, respectively. The 12 months mortality was 50 and $44 \%$ at pre and post intervention, respectively (Table 2). There was no difference in the mortality between men and women.

Endocarditis, spondylitis, and pneumonia were the most common manifestations related to SAB. During the pre intervention period, none of the seven patients with endocarditis was sent to cardiac surgery during hospital stay; however, four out of nine patients were sent to cardiac surgery at post intervention. A major part of patients with $\mathrm{SAB}$ had an unclear focus (Table 2).

The number of patients suffering from a nosocomial disease was $12(21 \%)$ at pre intervention and $24(24 \%)$ at post intervention $(p=0.63)$. The time from arrival at the hospital to the first antibiotic dosage administered (not including

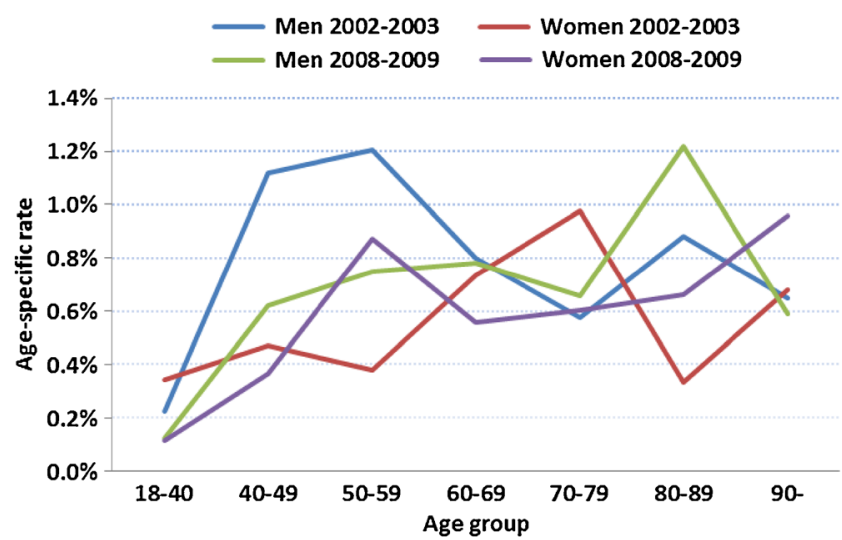

Fig. 1 Age-specific rates of cases with Staphylococcus aureus bacteremia (SAB) during the years 2002-2003 and 2008-2009

nosocomial disease) was, on average, $14 \mathrm{~h}$ (median $6 \mathrm{~h}$ ) at pre intervention and $11 \mathrm{~h}$ (median $4 \mathrm{~h}$ ) at post intervention $(p=0.38)$. The mean duration of intravenous antibiotic treatment was 13 days at pre intervention and 15.5 days at post intervention $(p=0.23)$. Cefuroxime was the most common antibiotic drug at the start of treatment pre intervention, given to $45(78 \%)$ patients, and cefotaxime at post intervention, given to 25 patients $(25 \%)$. Cloxacillin was given to 79 patients $(50 \%)$ during treatment, but was used as the first antibiotic in only 12 cases ( $8 \%$ ). Oral antibiotics after a period of intravenous antibiotics were given to 48 patients $(83 \%)$ pre intervention and to 67 patients $(67 \%)$ post intervention $(p=0.03)$.

At pre intervention, 45 out of 58 patients $(78 \%)$ and at post intervention, 69 out of 100 patients $(69 \%)$ were referred to a specialist in infectious diseases $(p=0.25)$, and $19(33 \%)$ and $26(26 \%)$ at pre and post intervention, respectively, were treated at the infectious disease department $(p=0.36)$. Hospitalization was delayed (median 1 day) for 28 and $39 \%$ $(p=0.15)$ of patients at pre and post intervention, respectively. The mortality rate of these patients did not differ from the mortality of patients immediately hospitalized at the first medical contact $(p=0.35)$. The number of SAB patients treated at the intensive care unit increased from four $(7 \%)$ patients
Table 1 Annual incidence of Staphylococcus aureus bacteremia (SAB)

${ }^{\text {a }}$ Significant if $p$-value $\leq 0.05$

${ }^{\mathrm{b}}$ Difference between pre and post intervention

${ }^{\mathrm{c}}$ Compares the years 2002-2003 with 2008-2009

${ }^{\mathrm{d}}$ Compares men and women for each period of time

\begin{tabular}{|c|c|c|c|c|c|}
\hline & $2002-2003$ & $p$-Value ${ }^{\mathrm{a}}$ & $2008-2009$ & $p$-Value ${ }^{\mathrm{a}}$ & $\%$ \\
\hline Blood cultures & 8,616 & & 14,842 & $<0.0001^{\mathrm{c}}$ & +72 \\
\hline Men & 4,538 & & 8,224 & & +81 \\
\hline Women & 4,078 & $<0.0001^{\mathrm{d}}$ & 6,618 & $<0.0001^{\mathrm{d}}$ & +62 \\
\hline $\begin{array}{l}\text { Number of positive blood } \\
\text { cultures with } S \text {. aureus }\end{array}$ & 161 & & 218 & $0.019^{\mathrm{c}}$ & +35 \\
\hline SAB & 58 & & 100 & $0.0037^{\mathrm{c}}$ & +72 \\
\hline Men & 35 & & 62 & & +77 \\
\hline Women & 23 & $0.071^{\mathrm{d}}$ & 38 & $0.009^{\mathrm{d}}$ & +65 \\
\hline Annual incidence per 100,000 & 28 & & 45 & $0.046^{\mathrm{c}}$ & +61 \\
\hline
\end{tabular}


Table 2 All-cause mortality after Staphylococcus aureus bacteremia (SAB) and clinical manifestations at pre and post intervention
${ }^{\text {a }}$ Significant if $p$-value $\leq 0.05$

${ }^{\mathrm{b}}$ Manifestations may occur more than once in an individual

\begin{tabular}{|c|c|c|c|c|c|c|}
\hline & \multicolumn{2}{|c|}{ Years 2002-2003 (\%) } & \multirow[t]{2}{*}{$p$-Value ${ }^{\mathrm{a}}$} & \multicolumn{2}{|c|}{ Years 2008-2009 (\%) } & \multirow[t]{2}{*}{$p$-Value ${ }^{\mathrm{a}}$} \\
\hline & Men & Women & & Men & Women & \\
\hline \multicolumn{7}{|l|}{ Mortality } \\
\hline During hospital stay & $6(17.1)$ & $2(8.7)$ & 0.36 & $11(17.7)$ & $7(18.4)$ & 0.93 \\
\hline $\begin{array}{l}\text { Within } 3 \text { months } \\
\text { after discharge }\end{array}$ & $5(14.3)$ & $4(17.4)$ & 0.75 & $8(12.9)$ & $4(10.5)$ & 0.72 \\
\hline $\begin{array}{l}\text { During } 3-12 \text { months } \\
\text { after discharge }\end{array}$ & $9(25.7)$ & $3(13.0)$ & 0.24 & $11(17.7)$ & $3(7.9)$ & 0.17 \\
\hline Total & $20(57.1)$ & $9(39.1)$ & 0.18 & $30(48.3)$ & $14(36.8)$ & 0.25 \\
\hline Clinical manifestations ${ }^{\mathrm{b}}$ & \multicolumn{3}{|l|}{ Total } & \multicolumn{3}{|l|}{ Total } \\
\hline Skin or soft tissue injury & \multicolumn{3}{|l|}{$8(14)$} & \multicolumn{2}{|l|}{$29(29)$} & 0.03 \\
\hline Endocarditis & \multicolumn{3}{|l|}{$7(12)$} & \multicolumn{2}{|l|}{$9(9)$} & 0.54 \\
\hline Spondylitis & \multicolumn{3}{|l|}{$4(7)$} & \multicolumn{2}{|l|}{$11(11)$} & 0.4 \\
\hline Osteitis & \multicolumn{3}{|l|}{$4(7)$} & \multicolumn{2}{|l|}{$6(6)$} & 0.82 \\
\hline Arthritis & \multicolumn{3}{|l|}{$3(5)$} & \multicolumn{2}{|l|}{$7(6)$} & 0.65 \\
\hline Prosthetic infection & \multicolumn{3}{|l|}{$1(2)$} & \multicolumn{2}{|l|}{$2(2)$} & 0.90 \\
\hline Pneumonia & \multicolumn{3}{|l|}{$1(2)$} & \multicolumn{2}{|l|}{$16(16)$} & 0.0052 \\
\hline Unclear focus & \multicolumn{3}{|l|}{$23(40)$} & \multicolumn{2}{|l|}{$25(25)$} & 0.054 \\
\hline
\end{tabular}

pre intervention to $22(22 \%)$ patients post intervention $(p=0.02)$. Heart ultrasound examination was performed on 30 patients at pre intervention (52\%) and on 76 patients at post intervention (76\%) $(p=0.0017)$. The average length of hospital stay was 20 days at pre intervention and 24 days at post intervention $(p=0.81)$.

\section{spa typing}

In total, 163 out of 173 isolates were available for spa typing from 148 of the 158 patients. During pre and post intervention, 42 and 58 different spa types were detected, respectively. The BURP cluster analysis resulted in three dominating spa clonal complexes (CCs) (Fig. 2). These comprised 55 and $61 \%$ of all spa types at pre and post intervention, respectively. The three clusters were equally distributed within each time period and between the two periods. Minor differences in the spa type distribution between pre and post intervention were noted (Fig. 2).

A total of 12 patients suffered from 13 recurrences. All isolates were available for spa typing from both the primary infection and the recurrence. Eleven patients had relapses and two patients had a reinfection. The spa types seen in the reinfected patients were $\mathrm{t} 9201 / \mathrm{t} 246$ and $\mathrm{t} 183 / \mathrm{t} 505$, respectively. Two patients had a dual infection, i.e., $S$. aureus of different spa types isolated from the same bottle. These spa types were t021/t037 (resistant to fusidic acid) and t084/t362, respectively. No spa type was associated with increased risk of death or clinical manifestations (Table 2).

\section{AST}

All of the 171 out of $173 \mathrm{~S}$. aureus isolates analyzed were sensitive to clindamycin, tobramycin, trimethoprim-sulfamethoxazole, vancomycin, erythromycin, rifampicin, netilmicin, linezolid, quinolones meropenem, cefadroxil, and cefuroxime. Fusidic acid AST resulted in 14 resistant isolates pre intervention compared to one resistant isolate post intervention. No MRSA isolates were detected.

\section{PVL testing}

No PVL-positive isolates were found.

\section{Discussion}

The main finding of this study is that the implementation of a quality improvement program, aiming at the early detection of patients with severe sepsis, resulted in a $61 \%$ increase in the annual incidence of SAB, which was sustained at the 3 years follow up. This indicates that a substantial number of SABs remained undetected before the intervention. The greatest increase in SAB was seen in men, resulting in a significantly higher $\mathrm{SAB}$ rate in men than in women at post intervention, which is in agreement with other reports [12, 13]. Furthermore, no spa types correlated to increased risk of death or specific clinical manifestations.

Our pre intervention SAB incidence figures are in line with the results of Jacobsson et al. [14] performed in geographic 


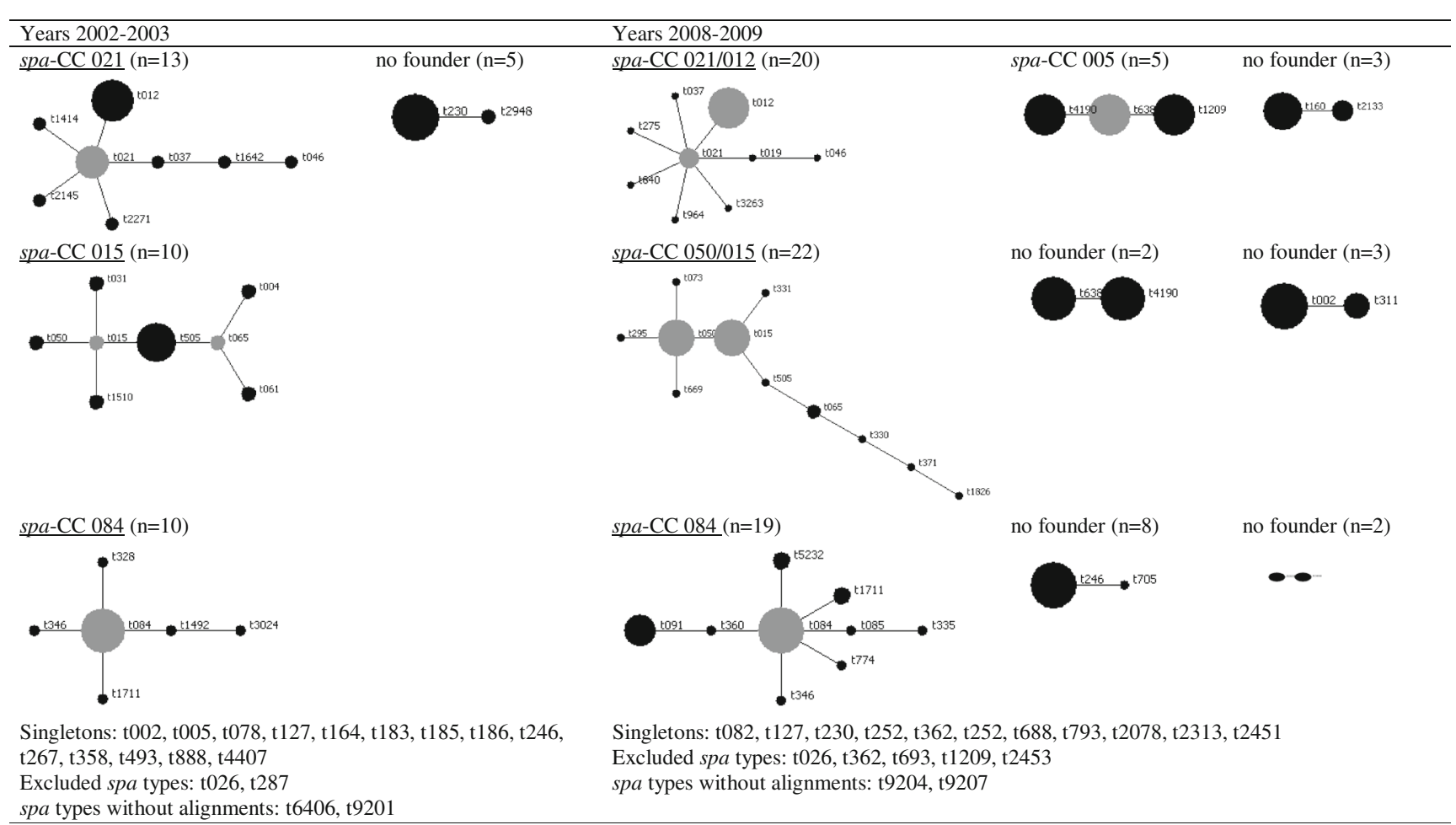

Fig. 2 Based upon repeat pattern (BURP) clustering of 60 isolates from years 2002-2003 and 103 isolates from years 2008-2009 based on spa types. The spa type with the highest founder score is defined as the founder of the cluster and is given the cluster its name. Subfounders are the spa types with the second highest founder score. Founders and subfounders are marked in gray. Each dot in a cluster represents a unique spa type and the diameter of the dot represents the quantity of the spa type

have compensated for this and, thus, better reflects the true SAB incidence in the population.

The mortality rates during hospital stay and after 12 months did not change significantly between pre and post intervention. Hence, the SAB patients detected through the quality

Table 3 The most common isolates from other studies in comparison with the 14 most common spa types in this study

\begin{tabular}{|c|c|c|c|c|c|}
\hline spa types & $\begin{array}{l}\text { This study, } n=163 \\
\text { No.(\%) of isolates }\end{array}$ & $\begin{array}{l}\text { Aamot et al. [18], } n=353 \\
\text { No. (\%) of isolates }\end{array}$ & $\begin{array}{l}\text { Sangvik et al. [26], } n=1,113 \\
\text { No. (\%) of isolates }\end{array}$ & $\begin{array}{l}\text { Matussek et al. [19], } n=160 \\
\text { No.(\%) of isolates }\end{array}$ & $\begin{array}{l}\text { Mernelius et al. [20], } n=1,635 \\
\text { No. (\%) of isolates }\end{array}$ \\
\hline t012 & $13(8.0 \%)$ & $14(4.0 \%)$ & $94(8.4 \%)$ & $20(12.5 \%)$ & $65(4.0 \%)$ \\
\hline $\mathrm{t} 084$ & $11(6.7 \%)$ & $46(13.0 \%)$ & $84(7.6 \%)$ & & $104(6.4 \%)$ \\
\hline $\mathrm{t} 246$ & $8(4.9 \%)$ & & & & \\
\hline $\mathrm{t} 021$ & $7(4.3 \%)$ & & $42(3.8 \%)$ & $10(6.25 \%)$ & $42(2.6 \%)$ \\
\hline $\mathrm{t} 050$ & $7(4.3 \%)$ & & & & \\
\hline t015 & $7(4.3 \%)$ & & $38(3.4 \%)$ & $10(6.25 \%)$ & $50(3.1 \%)$ \\
\hline $\mathrm{t} 230$ & $6(3.7 \%)$ & & & & \\
\hline t091 & $4(2.5 \%)$ & & & & \\
\hline $\mathrm{t} 505$ & $4(2.5 \%)$ & & & & \\
\hline t065 & $3(1.8 \%)$ & $15(4.2 \%)$ & $55(4.9 \%)$ & & \\
\hline t026 & $3(1.8 \%)$ & & & & \\
\hline $\mathrm{t} 1711$ & $3(1.8 \%)$ & & & & \\
\hline t002 & $3(1.8 \%)$ & $18(5.1 \%)$ & $30(2.7 \%)$ & & $41(2.5 \%)$ \\
\hline t005 & $3(1.8 \%)$ & & & & \\
\hline
\end{tabular}


improvement program seems to have comparable mortality as the pre intervention patients. In contrast to a recent report by Jacobson and Nasic showing increased mortality in women, we show similar mortality rates between men and women [16].

The numbers of clinical manifestations are too few to draw significant conclusions. However, one serious and common manifestation was infective endocarditis [17], seen in approximately $10 \%$ of the patients in this study. Notably, although we observed an increase in transesophageal or transthoracic echocardiography between pre and post intervention, the rate of endocarditis remained stable. None of the patients with endocarditis were sent to cardiac surgery during pre intervention compared to four out of nine patients during post intervention, which may reflect modified treatment recommendations.

Among the other clinical manifestations, pneumonias and skin and soft tissue infections were higher at post intervention. Only the isolates from the patients suffering from pneumonia were PVL tested and none were positive. The relatively high proportion of pneumonias should, therefore, be interpreted with caution, since the study was performed retrospectively without an independent evaluation of the clinical diagnosis.

Between pre and post intervention, we found an average non-significant decrease of $3 \mathrm{~h}$ from admission to hospital to the start of antibiotic treatment and longer duration of intravenous treatment with antibiotics.

Although the intervention program included a recommendation to contact an infectious disease specialist, no increase in referrals was noted. Moreover, delay to hospitalization was not decreased.

We found a large diversity of spa types and stability over time for common types, which confirm recent Norwegian findings [18]. The common spa types were also found in recent reports on healthy individuals from the same geographic area [19-21], as in our study (Table 3). This is in agreement with the finding that a successful colonizing strain can transform into a life-threatening human pathogen at any time [22-24]. Notably, spa type t084, frequently found during both pre and post intervention, could not be detected in the study by Matussek et al. [19] performed during the time for our pre intervention, but was later detected in healthy individuals [20]. This may indicate that this type was first established in clinical infections in our region. No specific spa type correlated to clinical manifestations or mortality, although a correlation to mortality was recently reported [18].

Relapse was more common than reinfection, and the two patients with reinfection had spa types of great difference. The two patients with dual infection, in contrast, had two closely related spa types, which may indicate genetic changes during infection.

A generally low rate of antibiotic resistance was observed and no MRSA was detected. The most distinguished resistance was to fusidic acid, noted in 14 isolates during pre intervention compared to one isolate during post intervention. This may reflect an outbreak of $S$. aureus fusidic acid resistance among Swedish children with bullous impetigo during the mid-1990s [25].

\section{Conclusions}

The strong recommendation to perform a blood culture prior to intravenous antibiotics resulted in an increased detection of Staphylococcus aureus bacteremia (SAB), especially in men. Hence, the SAB patients detected through the quality improvement program seems to have comparable mortality as the pre intervention patients. Specific spa types could not be correlated to clinical manifestations or mortality.

Acknowledgments This study was, in part, financed by Futurum - The Academy for Healthcare, Jönköping County Council.

Conflict of interest The authors confirm that they have no conflicts of interest.

Open Access This article is distributed under the terms of the Creative Commons Attribution License which permits any use, distribution, and reproduction in any medium, provided the original author(s) and the source are credited.

\section{References}

1. Laupland KB, Davies HD, Church DL, Louie TJ, Dool JS, Zygun DA, Doig CJ (2004) Bloodstream infection-associated sepsis and septic shock in critically ill adults: a population-based study. Infection 32:59-64

2. Rivers E, Nguyen B, Havstad S, Ressler J, Muzzin A, Knoblich B, Peterson E, Tomlanovich M; Early Goal-Directed Therapy Collaborative Group (2001) Early goal-directed therapy in the treatment of severe sepsis and septic shock. N Engl J Med 345:1368-1377

3. Kumar A, Roberts D, Wood KE, Light B, Parrillo JE, Sharma S, Suppes R, Feinstein D, Zanotti S, Taiberg L, Gurka D, Kumar A, Cheang M (2006) Duration of hypotension before initiation of effective antimicrobial therapy is the critical determinant of survival in human septic shock. Crit Care Med 34:1589-1596

4. Dellinger RP, Carlet JM, Masur H, Gerlach H, Calandra T, Cohen J, Gea-Banacloche J, Keh D, Marshall JC, Parker MM, Ramsay G, Zimmerman JL, Vincent JL, Levy MM; Surviving Sepsis Campaign Management Guidelines Committee (2004) Surviving Sepsis Campaign guidelines for management of severe sepsis and septic shock. Crit Care Med 32:858-873

5. Laupland KB, Lyytikäinen O, Søgaard M, Kennedy KJ, Knudsen JD, Ostergaard C, Galbraith JC, Valiquette L, Jacobsson G, Collignon P, Schønheyder HC; International Bacteremia Surveillance Collaborative (2013) The changing epidemiology of Staphylococcus aureus bloodstream infection: a multinational population-based surveillance study. Clin Microbiol Infect 19:465-471

6. Paul M, Kariv G, Goldberg E, Raskin M, Shaked H, Hazzan R, Samra Z, Paghis D, Bishara J, Leibovici L (2010) Importance of appropriate empirical antibiotic therapy for methicillin-resistant Staphylococcus aureus bacteraemia. J Antimicrob Chemother 65: 2658-2665 
7. Harmsen D, Claus H, Witte W, Rothgänger J, Claus H, Turnwald D, Vogel U (2003) Typing of methicillin-resistant Staphylococcus aure$u s$ in a university hospital setting by using novel software for spa repeat determination and database management. J Clin Microbiol 41: 5442-5448

8. Bone RC, Balk RA, Cerra FB, Dellinger RP, Fein AM, Knaus WA, Schein RM, Sibbald WJ (1992) Definitions for sepsis and organ failure and guidelines for the use of innovative therapies in sepsis. The ACCP/SCCM Consensus Conference Committee. American College of Chest Physicians/Society of Critical Care Medicine. Chest 101:1644-1655

9. van Belkum A, Tassios PT, Dijkshoorn L, Haeggman S, Cookson B, Fry NK, Fussing V, Green J, Feil E, Gerner-Smidt P, Brisse S, Struelens M; European Society of Clinical Microbiology and Infectious Diseases (ESCMID) Study Group on Epidemiological Markers (ESGEM) (2007) Guidelines for the validation and application of typing methods for use in bacterial epidemiology. Clin Microbiol Infect 13(Suppl 3):1-46

10. Mellmann A, Weniger T, Berssenbrügge C, Rothgänger J, Sammeth M, Stoye J, Harmsen D (2007) Based Upon Repeat Pattern (BURP): an algorithm to characterize the long-term evolution of Staphylococcus aureus populations based on spa polymorphisms. BMC Microbiol 7:98

11. Lina G, Piémont Y, Godail-Gamot F, Bes M, Peter MO, Gauduchon V, Vandenesch F, Etienne J (1999) Involvement of Panton-Valentine leukocidin-producing Staphylococcus aureus in primary skin infections and pneumonia. Clin Infect Dis 29:1128-1132

12. Big C, Malani PN (2010) Staphylococcus aureus bloodstream infections in older adults: clinical outcomes and risk factors for in-hospital mortality. J Am Geriatr Soc 58:300-305

13. Bassetti M, Trecarichi EM, Mesini A, Spanu T, Giacobbe DR, Rossi M, Shenone E, Pascale GD, Molinari MP, Cauda R, Viscoli C, Tumbarello M (2012) Risk factors and mortality of healthcareassociated and community-acquired Staphylococcus aureus bacteraemia. Clin Microbiol Infect 18:862-869

14. Jacobsson G, Dashti S, Wahlberg T, Andersson R (2007) The epidemiology of and risk factors for invasive Staphylococcus aureus infections in western Sweden. Scand J Infect Dis 39:6-13

15. Jacobsson G, Gustafsson E, Andersson R (2008) Outcome for invasive Staphylococcus aureus infections. Eur J Clin Microbiol Infect Dis 27:839-848

16. Jacobsson G, Nasic S (2012) Long-term outcome of invasive Staphylococcus aureus infections. Scand J Infect Dis 44:350-354

17. Murdoch DR, Corey GR, Hoen B, Miró JM, Fowler VG Jr, Bayer AS, Karchmer AW, Olaison L, Pappas PA, Moreillon P, Chambers ST, Chu VH, Falcó V, Holland DJ, Jones P, Klein JL, Raymond NJ, Read KM, Tripodi MF, Utili R, Wang A, Woods CW, Cabell CH;
International Collaboration on Endocarditis-Prospective Cohort Study (ICE-PCS) Investigators (2009) Clinical presentation, etiology, and outcome of infective endocarditis in the 21 st century: the International Collaboration on Endocarditis-Prospective Cohort Study. Arch Intern Med 169:463-473

18. Aamot HV, Blomfeldt A, Eskesen AN (2012) Genotyping of 353 Staphylococcus aureus bloodstream isolates collected between 2004 and 2009 at a Norwegian university hospital and potential associations with clinical parameters. J Clin Microbiol 50:31113114

19. Matussek A, Taipalensuu J, Einemo IM, Tiefenthal M, Löfgren S (2007) Transmission of Staphylococcus aureus from maternity unit staff members to newborns disclosed through spa typing. Am J Infect Control 35:122-125

20. Mernelius S, Löfgren S, Lindgren PE, Blomberg M, Olhager E, Gunnervik C, Lenrick R, Thrane MT, Isaksson B, Matussek A (2013) The effect of improved compliance with hygiene guidelines on transmission of Staphylococcus aureus to newborn infants: the Swedish Hygiene Intervention and Transmission of $S$ aureus study. Am J Infect Control 41:585-590

21. Mernelius S, Löfgren S, Lindgren PE, Matussek A (2013) The role of broth enrichment in Staphylococcus aureus cultivation and transmission from the throat to newborn infants: results from the Swedish hygiene intervention and transmission of $S$. aureus study. Eur J Clin Microbiol Infect Dis (in press). doi:10.1007/ s10096-013-1917-6

22. Lamers RP, Stinnett JW, Muthukrishnan G, Parkinson CL, Cole AM (2011) Evolutionary analyses of Staphylococcus aureus identify genetic relationships between nasal carriage and clinical isolates. PLoS One 6:e16426

23. Melles DC, Gorkink RF, Boelens HA, Snijders SV, Peeters JK, Moorhouse MJ, van der Spek PJ, van Leeuwen WB, Simons G, Verbrugh HA, van Belkum A (2004) Natural population dynamics and expansion of pathogenic clones of Staphylococcus aureus. J Clin Invest 114:1732-1740

24. von Eiff C, Becker K, Machka K, Stammer H, Peters G (2001) Nasal carriage as a source of Staphylococcus aureus bacteremia. Study Group. N Engl J Med 344:11-16

25. Osterlund A, Eden T, Olsson-Liljequist B, Haeggman S, Kahlmeter G; Swedish Study Group on Fusidic Acid-resistant Staphylococcus aureus (2002) Clonal spread among Swedish children of a Staphylococcus aureus strain resistant to fusidic acid. Scand J Infect Dis 34:729-734

26. Sangvik M, Olsen RS, Olsen K, Simonsen GS, Furberg AS, Sollid JU (2011) Age- and gender-associated Staphylococcus aureus spa types found among nasal carriers in a general population: the Tromso Staph and Skin Study. J Clin Microbiol 49:4213-4218 